\title{
CONSIDÉRATIONS SUR QUELQUES ASPECTS DE LA MORPHOLOGIE ET DE L'ORGANISATION GÉNÉRALE DE L'APPAREIL MAMIMAIRE
}

\author{
par \\ E. BOURDELLE \\ Professeur au Muséum National d'Histoire Naturelle.
}

Les glandes mammaires avec leurs vaisseaux sanguins ou lymphatiques, avec leurs nerfs, avec les dépendances de la peau qui leur sont annexées sous forme de sacs d'enveloppe, de mamelons ou de tétines, constituent un véritable appareil mammaire auquel est dévolue la fonction très spéciale et bien définie de la lactation. Cette fonction est certes étroitement solidaire de la fonction de génération; elle doit être cependant distinguée de celle-ci, car, si elle en est le complément ordinairement utile, elle n'est pas toujours absolument indispensable et elle est susceptible d'exister, à titre anormal il est vrai, ailleurs que chez les femelles. On peut considérer toutefois qu'elle constitue l'attribut physiologique le plus caractéristique des mammifères, voire peut-être, le plus général. L'appareil mammaire n'existe pas en effet, même sous une forme qui puisse le rappeler, dans aucune autre classe de vertébrés ; il n'appartient qu'à celle des mammifères. D'autre part, cet appareil n'est pas seulement l'apanage des femelles, il est presque toujours représenté chez les mâles par des éléments plus ou moins évidents; il est même susceptible dans certains cas, chez ces derniers, de produire du lait véritable.

L'appareil mammaire, en principe symétrique, est essentiellement constitué par une ou plusieurs glandes latérales disposées de part et d'autre du plan médian du corps et ainsi associées par paires. Ces glandes sont desservies chacune par des canaux lactifères excréteurs en grand nombre. Les canaux s'ouvrent, isolément ou en commun, sur un champ cutané, exceptionnellement déprimé en poche mammaire, le plus souvent projeté en saillie plus ou moins accusée sous forme de mamelon ou tétine. Ces dépendances cutanées de I'appareil mammaire sont la traduction extérieure la plus évidente de cet appareil et, ainsi que cela arrive souvent en matière de morphologie animale, la partie directement visible de l'organe est prise pour le tout, désignée sous son nom et sert à l'apprécier. C'est ainsi que les zoologistes ne comptent guère les mamelles dans les différentes espèces que par le nombre des tétines ou mamelons qui les décèlent. Peut-être y a-t-il là des causes graves d'erreur dans l'appréciation des faits, surtout si l'on tient compte que poches mammaires, mamelons ou tétines n'ont pas toujours la même signification morphologique.

(I) Paru dans le Volume jubilaire du Professeur Ch. Porcher. 
La poche mammaire proprement dite n'est que la dépression d'un champ glandulaire en surface qui, à l'époque de la reproduction, s'entoure d'un léger bourrelet cutané et se creuse faiblement (Echidné). Cette dépression temporaire peut s'accuser jusqu'à constituer un véritable sinus galactophore, au fond duquel s'ouvrent les conduits lactifères, et ce sinus peut prendre un caractère permanent (Halmaturus). La poehe mammaire ainsi formée ne doit pas être confondue avec le marsupium ou poche marsupiale. Celle-ci est une formation cutanée indépendante de la précédente, destinée à recevoir le jeune. Elle se forme à proximité de la poche mammaire, ouverte en avant ou en arrière de celle-ci ou l'englobant complètement suivant les espèces. Dans l'appréciation extérieure de l'appareil mammaire chez les Marsupiaux, il y a done à faire tout d'abord la part du marsupium pour apprécier ce qui revient à la poche mammaire.

Les formations cutanées en saillies del'appareil mammaire n'ont pas toujours la même signification, malgréles apparences morphologiques.

Dans certains cas, le plus fréquent semble-t-il, la tétine n'est que l'exagération de la poche mammaire en un sinus galactophore très étiré, par accroissement et surélévation du bourrelet périphérique qui enserrait la dépression primitive du champ glandulaire cutané. Dans ce cas, la tétine n'est percée à son extrémité que d'un seul orifice, celui du sinus galactophore, qui a pris l'aspect d'un long conduit galactophore, mais au fond duquel s'ouvrent les conduits lactifères des glandes en nombre multiple.

Dans d'autres cas, le champ glandulaire cutané sur lequel s'ouvrent les canaux lactifères s'est progressivement élevé et étiré au lieu de se déprimer et des canaux lactifères multiples viennent s'ouvrir ainsi à l'extrémité d'un mamelon plus ou moins surélevé (Carnivores, Primates). On voit ainsi, malgré les apparences morphologiques extérieures de similitude, les différences profondes qui séparent dans leur organisation les mamelons ou tétines des divers mammifères. Ces différences vont d'ailleurs beaucoup plus loin encore quand on envisage la disposition de ces parties externes de l'appareil mammaire chez les Marsupiaux, par exemple. Chez certains de ces animaux (Phalangers, Péramèles), la poche mammaire existe bien constituée, bien circonscrite, à proximité ou à l'intérieur de la poche marsupiale. A son fond, les canaux lactifères convergent sur une papille en mamelon rudimentaire. Dans d'autres espèces (Didelphes), ce mamelon accuse son développement et à l'époque de la lactation, il s'évagine même complètement de la poche mammaire pour se présenter en saillie dans la poche marsupiale ou à proximité de son ouverture, à la façon d'une tétine ordinaire. Mais dans ce cas, on le voit, la tétine en saillie n'a plus ni la signification de la tétine du pis de la vache, par exemple, ni celle d'une tétine en mamelon ordinaire. 
Les difficultés relatives à l'interprétation des dispositions et des caractères extérieurs de l'appareil mammaire de certaines espèces ont été telles que le doute a persisté pendant longtemps, quant à la. place que ces espèces devaient occuper dans la classification zoologique et que les zoologistes ont été longs à se mettre d'accord à leur sujet. On ne voulait, en effet, considérer tout d'abord comme mammifères que les animaux pourvus de mamelons ou tétines bien caractérisés. C'est ainsi que pour les monotrèmes en particulier, avec l'Ornithorhynque (Platypus ou mieux Ornithorhyncus Anatinus), l'absence de tétines a laissé le plus grand doute pendant longtemps sur la place à assigner à cet animal dans la classification. Alors que Home donnait déjà en 1802 une bonne description générale de cet animal, il ne faisait aucune allusion à ses glandes mammaires. TIEDEMAN, en 1808, LAMARCK, en 1809, ILLIGER, en 1812, restaient dans la même ignorance et n'osaient classer l'Ornithorhynque parmi les mammifères. Blainville lui-même, en 1812, quoique moins affirmatif, en fit avec l'Echidné l'ordre distinct des Ornithodelphes. Etienne Geoffroy SaInt-Hillitre et Latreille partageaient la même manière de voir, alors que Cuvier inclinait nettement vers l'opinion contraire. Ce n'est qu'en 1827 qu'Isidore Geoftroy SAINTHilaire fit entrer l'Ornithorhynque dans les mammifères et, presque en même temps, Mecker découvrait les glandes mammaires et en donnait une première description.

Chez ces animaux, les canaux lactifères s'ouvrent par un certain. nombre de pores sur une petite surface de la peau située de part et. d'autre du plan médian du corps à la face inférieure de l'abdomen, en avant du cloaque. Il n'y a pas de mamelons caractérisés. A leur place existe une touffe de poils qui en tient lieu. A la fin de la saison des naissances, les glandes mammaires deviennent si petites qu'elles sont à peine perceptibles et au moment où le jeune sort de l'œuf elles ne donnent pas encore de lait. Cet état d'inactivité persiste encore après l'éclosion et ce n'est qu'au bout d'un certain temps que la. sécrétion s'établit. Celle-ci est une véritable excrétion qui se fait en dehors de toute action de succion du jeune qui, à défaut de mamelon, profite du liquide qui s'écoule sur le pinceau de poils de l'aire glandulaire. Le lait ainsi sécrété paraît d'une nature particulière et, sous ce rapport comme sous celui de la nature même des glandes mammaires et de la disposition générale, l'appareil mammaire de l'Ornithorhynque et des Monotrèmes est bien différent de celui des autres mammifères.

$$
* * *
$$

Des dispositions déjà nettement différentes de l'appareil mammaire s'observent chez les Marsupiaux. Des mamelons distincts 
existent en effet chez ces animaux, soit à demi-inclus chacun dans la poche mammaire au fond de laquelle ils se sont développés, soit totalement évaginés de cette fosse, qui semble alors avoir disparu. Les poches mammaires ou les mamelons qui se sont formés à leurs dépens se présentent comme des organes complètement indépendants de la poche marsupiale. Celle-ci est d'ailleurs parfois à l'état rudimentaire et elle fait même totalement défaut chez certains Marsupiaux (Dasyures). Si cette poche marsupiale n'appartient pas en propre à l'appareil mammaire, elle constitue toutefois une annexe intéressante de cet appareil, puisqu'elle soutient le jeune, fixé d'autre part par sa bouche à l'une des tétines de la mère. Dans les espèces qui possèdent une poche marsupiale bien développée, la poche existe à l'état rudimentaire chez les mâles de la même espèce, comme cela arrive chez Notoryctes Typhlops, par exemple. Le nombre des mamelles traduites par les tétines est variable suivant les espèces de Marsupiaux considérés. Il est le plus souvent assez élevé, rarement très réduit, mais le degré d'imperfection ou de développement des tétines, suivant les sujets, parfois très différents par leur âge et l'époque saisonnière à laquelle ils ont été examinés, a certainement été la cause d'erreurs dans le décompte des mamelles et cela permet d'expliquer les divergences des auteurs. C'est ainsi que si l'on attribue ordinairement quatre mamelles au Myrmecobius, WATERHOUSE en compte huit chez cet animal et que LECHE, de son côté, ne lui en accorde que cinq. La présence des tétines dans l'appareil mammaire des Marsupiaux constitue plus une adaptation qu'un perfectionnement à un mode nouveau de sécrétion du lait par succion de la part du jeune. Cette modalité nouvelle ne nécessite pas seulement une organisation spéciale des voies lactifères; elle comporte une constitution particulière de la glande mammaire, qui se présente bien différente dans sa structure, et même par le produit de la sécrétion, de celle des Monotrèmes. Mais si l'appareil mammaire des Marsupiaux se présente dans sa nature et dans son organisation nettement différenciée, en progrès sur celui des Monotrèmes, il ne peut fournir dans ses dispositions extérieures les caractères morphologiques de spécification précis qu'on trouve dans d'autres appareils.

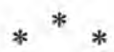

Les Monotrèmes et les Marsupiaux ne sont pas les seuls mammifères qui possèdent une poche mammaire plus ou moins bien caractérisée. Celle-ci se retrouve en effet, bien développée, chez les Cétacés, et parfaitement adaptée aux conditions particulières d'existence de ces animaux. Cette poche forme une cavité nettement définie qui s'ouvre par une fente de chaque côté de la vulve. A l'intérieur de la 
cavité et à son fond existe un mamelon en saillie bien détachée, à l'extrémité duquel s'ouvrent les orifices lactifères en nombre multiple. On retrouve en somme chez les Cétacés des dispositions analogues à celles qu'on observe chez certains Marsupiaux, c'est-à-dire' un véritable mamelon inclus dans une poche mammaire dont la disposition est manifestement en rapport avec les besoins particuliers: du jeune,

$$
*^{*} *
$$

Chez les animaux des groupes autres queles Monotrèmes, les Marsupiaux et les Cétacés, la présence de tétines ou de mamelons de formes et de dimensions variables, affirme toujours d'une façon assez évidente la position et le nombre des mamelles dans les différentes espèces. Cependant, des difficultés d'interprétation se présentent dans bien des cas et mettent les observateurs en désaccord. Les caractères que fournissent les mamelles, en vue de la spécification zoologique des mammifères, n'ont done pas toujours toute la valeur désirable.

En ce qui concerne la position des mamelles, on observe des dispositions très différentes. D'une façon générale cependant, les mamelles occupent une position ventrale de part et d'autre du plan médian sur le thorax, l'abdomen ou le bassin et, suivant le cas, on les dit thoraciques ou pectorales, abdominales, inguinales ou pelviennes. Dans certaines espèces, celles qui sont manifestement les plus prolifiques et les plus fécondes (Porc, Hérisson, Lapin, Lièvre, Agouti), elles s'allongent en deux traînées glandulaires qui paraissent continues sur l'ensemble de ces régions. Dans d'autres, on les voit se localiser sur la région thoracique en mamelles exclusivement pectorales (Homme, Singe, la plupart des Chiroptères, nombre d'Edentés : Tatous, Unau, Tamanoir, Eléphant, Siréniens). Chez d'autres espèces (Carnivores), elles se disposent dans la région abdominale, débordant, quand leur nombre augmente, sur la région thoracique ou sur la région inguinale (Insectivores, Rongeurs). Chez d'autres enfin (Equidés, Bovins, Caméliens), elles se localisent dans les régions inguinales, plus ou moins enfoncées dans le pli de l'aine. Il arrive même chez le Cachicanne (Dasypus Novencinctus) qu'on trouve à la fois une paire de mamelles pectorales et une paire de mamelles inguinales. Exceptionnellement chez certaines espèces, les mamelles émigrent de ces régions ventrales du thorax et de l'abdomen pour prendre une situation beaucoup plus périphérique. C'est ainsi que chez les Cétacés, on les trouve de chaque côté de la vulve; que chez certaines Chauves-souris, comme les grandes Roussettes, les mamelles sont axillaires; que chez la Viscache, elles sont placées sur les côtés de la poitrine, près de la face dorsale du corps, qu'elles accusent encore cette position dorsale chez le Ragondin (Myopotamus 
Coypu). Il s'agit là, bien entendu, de dispositions normales dans la situation des mamelles, mais on sait qu'anormalement on peut trouver des mamelles surnuméraires de caractère hétérotopique en des points différents et parfois très éloignés des lieux ordinaires de développement des mamelles dans une espèce déterminée. C'est ainsi que dans l'espèce humaine, on en a signalé dans l'aisselle, dans l'aine, sur l'épaule, le dos et jusque sur la cuisse et que parfois on a même pu noter la tendance héréditaire de certaines de ces anomalies.

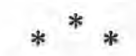

Depuis longtemps les zoologistes ont cherché à noter le nombre de mamelles dans les diverses espèces et, en même temps que les différences très importantes entre les groupes, ils ont observé de fréquentes variations individuelles, parfois pleines d'intérêt.

Le nombre de mamelles, en rapport assez étroit, semble-t-il, avec la fécondité de l'espèce, se réduit d'autre part dans les groupes les plus élevés de l'échelle zoologique ou dans les espèces de grande taille. C'est ainsi qu'il n'existe qu'une paire de mamelles chez l'Homme, les Singes, l'Hippopotame, le Tapir, les Cétacés, et chez la plupart des Lémuriens et des Chiroptères. Nombre de Ruminants, Caprins ou Antilopes, n'offrent aussi qu'une paire de mamelles, mais, d'une façon générale, la plupart des Ruminants, Bovins et Caméliens, possèdent deux paires de mamelles inguinales. Parmi les Carnivores, on compte ordinairement deux paires de mamelles chez le Lion, la Panthère, la Genette, la Loutre; trois paires chez l'Ours, le Puma, le Raton, le Coati, le Blaireau ; quatre paires chez le Chat, le Serval ; quatre à cinq paires chez le Chien et nombre de Canidés. Cinq paires existent chez le Hérisson, le Lapin; cinq à six paires chez le Porc; six et même sept paires chez l'Agouti et d'autres Rongeurs. Dans un même groupe, il y a souvent des différences importantes quant au nombre des mamelles. Nous en avons déjà noté quelques-unes chez les Ruminants et chez les Carnivores. Chez les Lémuriens, qui n'ont ordinairement qu'une paire de mamelles, comme les Makis, d'autres, tels que les Loris, en possèdent quatre.

Chez les Rongeurs, où le nombre des mamelles est ordinairement très élevé, il est des espèces, comme le Paca, le Castor, la Gerboise, et d'autres, comme le Cochon d'Inde, le Hamster, qui n'en ont même qu'une. Des faits de même ordre peuvent être notés chez les Chiroptères, chez les Insectivores, qui montrent, quant au nombre des éléments tout au moins, les plus grandes variations dans l'organisation de l'appareil mammaire.

Ces variations numériques dans le nombre des mamelles atteignent fréquemment les individus eux-mêmes, si bien qu'il est parfois 
difficile d'assigner un nombre absolument fixe de mamelles à certaines espèces ou races animales. C'est ainsi que, comme nous l'avons déjà fait remarquer, on trouve, suivant les races ou les sujets, cinq ou six paires de mamelles chez le Porc, quatre ou einq paires chez le Chien, trois ou quatre chez le Chat. Il peut même arriver qu'il y ait inégalité dans le nombre des mamelles des deux séries latérales et qu'une certaine asymétrie soit observée dans la disposition de l'appareil mammaire. Cette asymétrie serait due, soit à l'apparition d'une mamelle supplémentaire d'un côté, soit à la disparition d'un seul côté encore de l'une des mamelles normales.

On peut observer enfin l'apparition d'une paire complète de mamelles supplémentaires s'adjoignant au système des mamelles normales. Des faits de ce genre sont assez souvent constatés chez la Brebis et chez la Chèvre, ainsi que chez la Vache. Mais alors que chez cette dernière les mamelles supplémentaires se développent en arrière des mamelles normales, chez les premières elles n'apparaissent jamais qu'en avant, et elles ont sans doute, de ce fait et dans ce cas, une signification atavique qu'on ne saurait leur attribuer dans l'autre.

Les faits de polymastie ou de polythélie, c'est-à-dire relatifs à l'augmentation du nombre des mamelles, sont depuis longtemps connus et bien étudiés. Ceux qui se rapportent à la diminution du nombre de ces organes ont certainement beaucoup moins retenu l'attention. Leur intérêt n'est pas moins grand cependant et, sans doute, permettaient-ils autant que la polythélie, d'expliquer les asymétries de l'appareil mammaire quant au nombre de ses éléments, ainsi que l'apparition de certaines dispositions symétriques normales. La diminution en nombre des mamelles, tout comme l'augmentation, peut se faire et se fait sans doute souvent en fin de série, soit en avant, soit en arrière de chaque ligne latérale de mamelles ou des deux lignes à la fois. Mais ainsi qu'Aposteleano l'a démontré en étudiant les relations lymphatiques ganglionnaires des mamelles des Carnivores, il arrive parfois que la variation numérique se fait chez les animaux par la disparition de l'un des éléments d'une paire moyenne de mamelles, Le fait est patent chez le Chien, par exemple. Chez les sujets porteurs de einq paires de mamelles normales, on constate que les deux paires antérieures ont toujours des relations lymphatiques avec les ganglions axillaires, que les deux paires postérieures ont exclusivement des relations avec les ganglions inguinaux et que la paire moyenne, la troisième, possède à la fois de chaque côté, des relations lymphatiques axillaires et inguinales. Chez les Chiennes qui n'offrent que quatre paires de mamelles, les deux paires antérieures ont gardé leurs relations axillaires, les deux paires postérieures leurs relations inguinales et, dans ce cas, il n'est pas de mamelles ayant à la fois des relations lymphatiques et axillaires. Il y a donc là une preuve anato- 
mique de la disparition d'éléments moyens de la mamelle dans le processus de réduction de l'appareil mammaire de Ia chienne. Sans doute, cette possibilité de variation par diminution de nombre, en milieu de série, qui s'oppose à la formule de variation en fin de cérie, trouvera-t-elle encore son application en vue de l'interprétation de dispositions normales et anormales de l'appareil mammaire dans d'autres espèces que les Carnivores, et aussi dans l'interprétation des faits qui se rapportent aux variations numériques des organes multiples et disposés en séries.

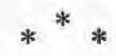

L'appareil mammaire n'est pas seulement un attribut exclusif des femelles. Il existe aussi d'une façon constante, mais dans un état plus ou moins rudimentaire, chez les mâles. Ceux-ci présentent en effet, presque tous, des traces de mamelles ou tout au moins de mamelons ou de tétines. C'est ainsi que chez les Primates mâles, on observe deux mamelles pectorales, parfois même assez bien constituées, C'est encore ainsi que chez le Verrat, chez le Chien, on observe deux traînées latérales de mamelles et de tétines, dans certains cas assez développées, qui rappellent à peu près exactement les mamelles en séries linéaires de la Truie et de la Chienne. Chez le Taureau, le scrotum porte en avant quatre petits tubercules, quelquefois deux seulement, vestiges des glandes mammaires de la Vache. Chez le Bouc et chez le Bélier, deux petits mamelons, en avant de la base du scrotum, souvent assez développés chez le Boue, ont encore la même signification. Chez le Chameau, ces mamelons, au nombre de quatre, sont situés en arrière du fourreau.

A l'état normal, les mamelles rudimentaires des mâles n'ont aucune activité. Ce n'est que par exception qu'elles sécrètent quelques gouttes de lait. Ce fait s'observe chez les enfants mâles à la naissance.

Mais l'appareil mammaire des mâles ne reste pas toujours à l'état rudimentaire : on a en effet observé et signalé depuis longtemps dans certaines espèces, des mâles porteurs de mamelles bien développées et susceptibles de produire normalement une certaine quantité de lait.

Chez l'Homme, le développement anormal des mamelles et de la sécrétion lactée a été signalé par RoBER, par HumboLdt, par FrankuIN, par Albers, par DuREglison. Dans certains cas, la sécrétion lactée aurait même été assez abondante pour pouvoir suffire à l'alimentation d'un nourrisson, ainsi que l'a relaté HuмвоLDT à propos d'un Indien du Pérou.

Chez les animaux, les cas de développement anormal des mamelles et de la sécrétion lactée des mâles ont été également souvent constatés dans diverses espèces. Nous avons déjà fait remarquer l'existence ou 
l'état très rudimentaire de la poche mammaire ou du marsupium chez certains Marsupiaux mâles adultes, les Thylacines, par exemple, ou chez certains foetus de Marsupiaux. Le Dasyure ourson a ainsi une poche jusqu'à l'âge de quatre mois. Chez les Ruminants domestiques, Bovins, Caprins, de nombreux cas de mamelles bien développées et actives ont été signalés. Un cas relatif à un Taureau de race Schwitz a été rapporté par Cornevin. Hrra, vétérinaire à Bombay, a depuis signalé le même fait chez les Taureaux émasculés. Chez le Bouc, les cas de développement anormal des mamelles et de sécrétion lactée sont encore plus fréquents que chez le Taureau et ont été souvent rapportés. Aristote relate le cas d'un Bouc qui fournissait abondamment du lait et qui transmit cette particularité à ses descendants ; Haler, Blumenthat, I. Ghoffroy Satnt-Hilatre, SchlossBERGER, ont rapporté des cas analogues; JoLY, un peu plus tard, a signalé la même particularité et, le premier, il a donné une analyse qui faisait ressortir la moins grande teneur en beurre du lait anormalement sécrété par le Bouc. A un certain moment, un Bouc à mamelles sécrétant du lait a vécu et a été conservé à la ménagerie du Muséum d'Histoire Naturelle à Paris.

Plus récemment encore, des observations nombreuses précises et complètes de même nature ont été rapportées. Tel est le cas de l'observation faite, en 1911, par BARBAZAN, vétérinaire à Maison Carrée, près Alger. Le Bouc qui en fit l'objet possédait en avant de la base des bourses, deux mamelles inguinales qui donnaient un lait excellent, épais et très sucré. Le sujet porteur de cette anomalie était âgé de deux ans. Il remplissait ses fonctions de géniteur d'une façon normale et il se laissait téter par deux chevrettes. Beaucoup de personnes eurent, paraît-il, l'occasion de goûter du lait de ce Bouc, qui fut vite l'objet d'une grande curiosité, si bien que BARBAzAN eut quelque peine à recueillir une quantité suffisante de liquide pour les analyses. Celles-ci, faites par le Professeur Porcher, de l'Ecole vétérinaire de Lyon, révélèrent dans le lait de ce boue les mêmes données que dans le lait de chèvre.

En 1912, en Algérie encore, Melus, vétérinaire à Souk-Ahras, a rapporté une observation de même nature que celle de BARBAZAN. Elle est relative à un Boue de race indigène âgé de trois ans qui, après avoir servi un troupeau comme étalon et engendré de nombreux produits, fut sacrifié à l'abattoir de Souk-Ahras. L'animal, d'aspect masculin et pourvu de tous les attributs de son sexe, présentait, immédiatement en avant des testicules, deux mamelles bien développées ayant sept à huit centimètres delong. Ces mamelles donnaient un lait normal en quantité assez importante pour que l'on puisse en extraire, journellement et à plusieurs reprises, la valeur d'un grand verre. La position des mamelles était à peu près normale. La dissec- 
tion de ces organes les a montrés constitués par un parenchyme glandulaire absolument semblable à celui des organes similaires de la Chèvre. On notait seulement l'importance des réservoirs galactophores, eu égard au volume du pis.

Un nouveau cas de développement des mamelles et de sécrétion lactée chez le Bouc a été rapporté dans The British Society Monthly Circular, en février 1922, par un lecteur de ce journal. Il était relatif à un sujet résultant d'un croisement de race anglo-nubienne et suisse, porteur de deux trayons analogues à ceux d'une Chèvre et qui donnait par jour, en dehors de la période de rut, un peu plus d'un demi-litre de lait. L'auteur de cette relation affirme avoir fait saillir avec succès ses chèvres par cet animal pendant qu'il était en lactation.

La même année, MAKalik, en Tchécoslovaquie, a observé l'anomalie sur un Bouc de la race Saanen, âgé de dix-huit mois, normalement développé et constitué comme mâle, sans trace d'hermaphrodisme. Ce Bouc avait fait ses premières saillies et avait déjà eu des descendants. Les mamelles et les tétines qu'il portait étaient serrées contre les bourses. Elles s'étaient développées spontanément, sans excitation locale préalable. Les tétines avaient déjà presque la longueur et l'épaisseur de celles d'une Chèvre normale. Ce Bouc produisait journellement 520 à $640 \mathrm{~cm}^{3}$ de lait de mêmeapparence que celui de la Chèvre, sans goût et sans odeur de Bouc. La composition chimique de ce produit, strictement analysé, ne révéla pas de très sensibles différences avec le lait normal de la Chèvre.

En 1925, enfin, le Général Stravascu, directeur des Services vétérinaires de l'Armée roumaine, a présenté à la Société centrale de Médecine vétérinaire, à Paris, un nouveau cas de développement anormal des mamelles et de lactation observé sur un Boue des Carpathes, au milieu d'un troupeau de Chèvres. Cet animal n'était ni un monstre ni un hermaphrodite. Agé de cinq ans, il avait fécondé, depuis l'année précédente, 60 chèvres, dont étaient nés 240 chevreaux mâles, tous en plein accroissement au moment de l'observation. Le earactère anormal des mamelles n'était apparu que sur deux de ses petits. Bien que le Bouc observé par le Général Stravescu n'eût jamais servi à l'allaitement et n'ait jamais été soumis à la mulsion, on s'était aperçu qu'il pouvait donner du lait. Le Général Stravescu put constater lui-même que ce lait n'avait pas le earactère d'un bon lait, mais plutôt celui d'un colostrum séreux.

Cet animal fut offert vivant à l'Ecole vétérinaire d'Alfort, aux fins d'observations, mais il mourut prématurément et ne put faire l'objet que d'une étude anatomique surle cadavre. Celle-ci, faite par nous-même, ne révéla rien d'anormal dans la constitution générale de l'appareil génital, en dehors de la présence des mamelles. Il n'y avait aucune trace d'hermaphrodisme externe, ni des voies génitales ni 
des voies glandulaires, sous réserve, cependant, de la possibilité de l'existence d'une glande génitale histologiquement mixte. On notait seulement la différence marquée de volume des deux testicules, le gauche étant sensiblement plus gros que le droit.

Les mamelles anormales formaient des masses coniques de 7 à 8 centimètres de long et de 4 centimètres d'épaisseur à la base, rappelant tout à fait, mais en plus petit, la forme des mamelles de la Chèvre. Elles étaient constituées par un parenchyme glandulaire très net, englobé dans un stroma conjonstif, de charpente assez serrée, le tout formant un ensemble dense et résistant. Chacune des masses présentait à la base une trace évidente de division en deux quartiers secondaires, l'un antérieur, l'autre postérieur. Peut-être pourrait-on voir dans cette division une manifestation, reste ou ébauche, des quatre mamelles que l'on observe normalement dans certaines espèces sauvages, voisines des Ovins et des Caprins, telles que les Oréas, les Tragelaphes, les Oryx et les Nemorrhèdes. Chacune des masses glandulaires était contenue dans une enveloppe cutanée fine et à peu près glabre, dont l'extrémité formait une tétine courte, mais très nette.

Le développement anormal des mamelles et l'apparition de la sécrétion lactée chez les mâles sont des faits incontestables et assez fréquents. Ils constituent une anomalie spontanée qui apparait sans aucune excitation artificielle de l'animal, surtout sans gymnastique fonctionnelle préalable, et dans laquelle l’hérédité ne semble pas jouer un rôle capital. Il s'agit bien là d'un développement anormal, à la fois anatomique et physiologique, de la glande, lequel ne doit pas être confondu, comme on a pu le croire, aveo une hypertrophie des éléments accessoires de l'organe, l'infiltration adipeuse par exemple. Si quelques cas de ee genre existent, ils sont tout à fait exceptionnels. Les mamelles rudimentaires et les ébauches glandulaires que présentent les mâles à l'état normal, permettent d'ailleurs d'expliquer, ou rendent, en tout cas, moins extraordinaire le développement de ces glandes.

L'activité physiologique de la sécrétion lactée des mamelles anormales des mâles pose à nouveau l'important problème des conditions intimes mêmes de cette sécrétion en général. En l'absence de tout hermaphrodisme chez les sujets qui offrent ce phénomène, on peut déduire : $1^{\circ}$ que, contrairement à ce que les Allemands PusH et Hansen affirment dans leur Traité de Zootechnie générale, l'aptitude laitière n'apparaît pas seulement chez les femelles et que les mâles peuvent, exceptionnellement, la présenter ; $2^{\circ}$ que la sécrétion lactée, contrairement à certaines théories, peut se développer en dehors de l'influence d'une hormone ovarienne ou d'une hormone spécifique fabriquée par l'utérus de la femelle au moment de la gestation. La 
possibilité de la sécrétion lactée chez les mâles montre que des influences autres que celles jusqu'à présent invoquées, doivent intervenir dans le mécanisme intime de cette sécrétion.

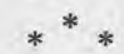

Les quelques aspects de la morphologie, de l'organisation ou des variations de l'appareil mammaire sur lesquels nous venons d'attirer l'attention, montrent l'intérêt qui s'attache à la connaissance et à l'étude de cet appareil. Certes on ne peut nier que depuis longtemps les zoologistes qui s'occupent des Vertébrés et en particulier des Mammifères, ont orienté leurs efforts de ce côté et que de nombreux faits sont déjà bien acquis. Il semble cependant que si les problèmes d'ordre morphologique sont à peu près résolus, d'importantes données ou conditions d'ordre biologique restent encore à établir. Sans doute ne pourra-t-on élucider ces dernières qu'en étroite liaison avec les premières, auxquelles elles restent intimement subordonnées ou qu'elles subordonnent, et là encore, comme en toutes les autres matières zoologiques, la physiologie apparaît étroitement solidaire de la morphologie.

\section{¡BIBLIOGRAPHIE.}

[1] Albers. Mastitis pubescentium virilis. Hover's Archiw für die gesammte medicis, 1844, 6, 272.

[2] Apostoleano. Contribution à l'étude du système lymphatique de la mamelle chez les carnivores domestiques. Thèse de Doctorat. Faculté de Méd., Paris, 1925.

[3] ARistote. Histoire des animaux. Traduetion de Camus, 1, 163.

[4] Barbazan. Mamelles en lactation chez le Bouc. Comptes rendus de la Société centrale de Médecine vétérinaire, 1911, 423.

[5] BEDdARD. The Cambridge Natural History : Mammalia, 1902.

[6] Buanc. Les anomalies de l'Homme et des Mammifères, 176.

[7] BlumenthaL. Verg. anat., 1805, 594.

[8] BRенм. Die Saügetiere, 1912.

[9] Bressou et Bourdelle. Etude des organes génitaux d'un Bouc à mamelles. Bull. de l'Acad. vét. de France, 1929, 2, 10, 354.

[10] BURrel. The Platypus. Australia, 1927.

[11] Chauvead, Arloing et Lesbre. Traité d'Anatomie comparée des animaux domestiques, $5^{\mathrm{e}}$ éd., 1903, Paris.

[12] Cornevin. Traité de Zootechnie générale, 292.

[13] Dechambre et Bourdelie. Le développement anormal des mamelles chez les mâles et en particulier chez le Bouc. Revue d'Histoire Naturelle, $1^{\text {re }}$ partie, 1928, 9, 10, 295.

[14] Dureglisson. Voir Carpenter : Principes of Human Physiology, 1853, 1061.

[15] Frankin. Narratives of a Journal to the Shores of the Polar Sea, 1819, 157. 
[16] Gegenbaur. Manuel d'Anatomie comparée, 1874, Paris.

[17] Isidore Geoffrox SATNT-Hilatre. Sur un Bouc lactifère. Comptes rendus. de l'Acad. des Sciences, 1852, 34, 386.

[18] Guinard. Précis de Tératologie, 1893, 235.

[19] HALleR. Elementa physiologia, 7, 2-18.

[20] Нбмвоцdт. Voyage aux régions équinoxiales du Nouveau Continent. Relation historique, $1,376$.

[21] LesBre. Traité de Tératologie de l'Homme et des animaux domestiques, 1927, Paris.

[22] Makalik. Le lait de Bouc. Le Lait, 1922, 340.

[23] Menuts. Revue vétérinaire, 1912, 215.

[24] Henri Mrune Edwards. Leçons sur la physiologie et l'anatomie comparée. Paris, 1870. Appareil mammaire, 9, 125. Londres.

[25] Oven. On the Anatomy of Vertebrates. Mammuals, I868, 3, 760.

[26] Porcher. Lactation chez le Bouc. Le Lait, 1922, 289.

[27] Roвer. Letter concerning a man who give suck to a child. Philos trans, $1742,41,461,813$.

[28] Sohlossberger. Analyse der Milch eine Bock. Ann. der Chimie und Pharm., $1844,5,431$.

[29] Stravescu. Comptes rendus de la Soc. cent. de Méd. vét., 1925, 80.

[30] Vialleton. Eléments de Morphologie des Vertébrés, 1911, Paris.

[31] Wiederschiev. Manuel d'Anatomie comparée des Vertébrés. Traduction de la deuxième édition allemande, par Moquin TANdon, 1890.

\title{
RECHERCHES SUR LA CASÉINE
}

\author{
par Mlle J. BRIGANDO
}

Docteur ès Sciences de l'Université de Lyon,

Chef de Travaux à l'Institut des Recherches Agronomiques.

(Suite.)

\section{RECHERCHES PERSONNELLES.} présure.

Ces recherches ont porté sur la caséine-acide et la caséine-

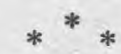

\section{CASÉINE-AGIDE.}

Notre but a été de déterminer les meilleures conditions dans lesquelles il faut se placer pour préparer une bonne caséine-acide, d'une teneur en cendres peu élevée, d'un bel aspect et d'une acidité faible.

Nous avons été amenée à examiner l'influence de la réaction du milieu, de l'agitation, de la température à laquelle la précipitation s'effectue, de la vitesse de l'addition de l'acide, de la concentration de ce dernier, du lavage. 